\section{Subcutaneous daratumumab in patients with relapsed or refractory multiple myeloma: part 2 of the open-label, multicenter, dose-escalation phase lb study (PAVO)}

\begin{abstract}
Jesus San-Miguel, ${ }^{1}$ Saad Z. Usmani, ${ }^{2}$ Maria-Victoria Mateos, ${ }^{3}$ Niels W.C.J. van de Donk, ${ }^{4}$ Jonathan L. Kaufman, ${ }^{5}$ Philippe Moreau, ${ }^{6}$ Albert Oriol, ${ }^{7}$ Torben Plesner, ${ }^{8}$ Lotfi Benboubker, ${ }^{9}$ Kevin Liu, ${ }^{10}$ Peter Hellemans, ${ }^{11}$ Tara Masterson, ${ }^{12}$ Pamela L. Clemens, ${ }^{12}$ Man Luo, ${ }^{12}$ Andrew Farnsworth, ${ }^{13}$ Hareth Nahi ${ }^{14}$ and Ajai Chari ${ }^{15}$
\end{abstract}

${ }^{1}$ Clínica Universidad de Navarra-CIMA, IDISNA, CIBERONC, Pamplona, Spain; ${ }^{2}$ Levine Cancer Institute/Atrium Health, Charlotte, NC, USA; ${ }^{3}$ University Hospital of

Salamanca/IBSAL, Salamanca, Spain; ${ }^{4}$ Amsterdam UMC, Vrije Universiteit Amsterdam, Department of Hematology, Amsterdam, the Netherlands; ${ }^{5}$ Winship Cancer Institute, Emory University, Atlanta, GA, USA; 'University Hospital of Nantes, Nantes, France; ${ }^{7}$ Institut Català d'Oncologia, HGTiP, Barcelona, Spain; ${ }^{8}$ Vejle Hospital and University of Southern Denmark, Vejle, Denmark; ' ${ }^{S}$ Service d'Hématologie et Thérapie Cellulaire, Hôpital Bretonneau, Centre Hospitalier Régional Universitaire (CHRU), Tours, France; ${ }^{10}$ Janssen Research \& Development, LLC, Raritan, NJ, USA; ${ }^{11}$ Janssen Research \& Development, Beerse, Belgium; ${ }^{12}$ Janssen Research \& Development, LLC, Spring House, PA, USA; ${ }^{13}$ Janssen Research \& Development, LLC, High Wycombe, UK; ${ }^{14}$ Karolinska Institute, Department of Medicine, Division of Hematology, Karolinska University Hospital at Huddinge, Stockholm, Sweden and ${ }^{15}$ Tisch Cancer Institute, Mount Sinai School of Medicine, New York, NY, USA

\section{ABSTRACT}

Tontrin ntravenous daratumumab is approved for the treatment of multiple myeloma. In part 1 of the PAVO study, a mix-and-deliver subcutaneous formulation of daratumumab with recombinant human hyaluronidase $\mathrm{PH} 20$ (rHuPH20) was well tolerated, with low rates of infusion-related reactions and an efficacy similar to that of intravenous daratumumab. Part 2 of PAVO evaluated a concentrated, pre-mixed co-formulation of daratumum$\mathrm{ab}$ and $\mathrm{rHuPH} 20$ (DARA SC). Patients who had received two or more prior lines of therapy, including a proteasome inhibitor and an immunomodulatory drug, were given daratumumab $(1,800 \mathrm{mg})$ and $\mathrm{rHuPH} 20(30,000 \mathrm{U})$ in $15 \mathrm{~mL}$ subcutaneously over 3 to 5 minutes as per the approved intravenous monotherapy dosing schedule. Primary endpoints were daratumumab trough concentration at the end of weekly dosing (just prior to the day 1 dose of cycle 3) and safety. Twenty-five patients were enrolled in PAVO part 2. DARA SC achieved daratumumab trough concentrations similar to or greater than those achieved with intravenous daratumumab $16 \mathrm{mg} / \mathrm{kg}$. The adverse event profile of DARA SC was consistent with that of intravenous daratumumab, with no new safety concerns and a lower infusion-related reaction rate. At a median follow-up of 14.2 months, the overall response rate was $52 \%$, the median duration of response was 15.7 months, and the median progression-free survival was 12.0 months. DARA SC 1,800 mg was well tolerated in relapsed/refractory multiple myeloma, with a low infusion-related reaction rate and reduced administration time. Daratumumab serum concentrations following DARA SC were consistent with those following intravenous dosing, and deep and durable responses were observed. Based on these results, ongoing studies are investigating DARA SC in the treatment of multiple myeloma and other conditions. (ClinicalTrials.gov identifier: NCT02519452).

\section{Introduction}

Daratumumab is a human IgGK monoclonal antibody targeting CD38 with a direct on-tumor ${ }^{1-4}$ and immunomodulatory ${ }^{5.7}$ mechanism of action. The direct ontumor actions of daratumumab are mediated by complement-dependent cytotoxi-
Ferrata Storti Foundation

Haematologica 2021

Volume 106(6):1725-1732

\section{Correspondence:}

JESUS SAN-MIGUEL

sanmiguel@unav.es

Received: November 26, 2019.

Accepted: April 29, 2020.

Pre-published: April 30, 2020.

https://doi.org/10.3324/haematol.2019.243790

(C)2021 Ferrata Storti Foundation

Material published in Haematologica is covered by copyright. All rights are reserved to the Ferrata Storti Foundation. Use of published material is allowed under the following terms and conditions:

https://creativecommons.org/licenses/by-nc/4.0/legalcode. Copies of published material are allowed for personal or internal use. Sharing published material for non-commercial purposes is subject to the following conditions:

https://creativecommons.org/licenses/by-nc/4.0/legalcode, sect. 3. Reproducing and sharing published material for commercial purposes is not allowed without permission in writing from the publisher. 
city, antibody-dependent cell-mediated cytotoxicity, antibody-dependent cellular phagocytosis, and apoptosis. ${ }^{1-4}$ The immunomodulatory actions of daratumumab lead to modulation of the tumor microenvironment, including the depletion of $\mathrm{CD}_{3} 8^{+}$immunosuppressive cells, which may explain the clonal expansion of cytotoxic $\mathrm{T}$ cells, increase in helper $\mathrm{T}$ cells, and increase in granzyme $\mathrm{B}^{+} \mathrm{CD} 8^{+} \mathrm{T}$ cells observed following exposure to this drug. ${ }^{5 \cdot 7}$

Intravenous (IV) daratumumab $16 \mathrm{mg} / \mathrm{kg}$ is approved as a monotherapy and in combination with bortezomib/dexamethasone, lenalidomide/dexamethasone, or pomalidomide/dexamethasone (in the USA) in patients with relapsed or refractory (RR) multiple myeloma (MM). ${ }^{8,9}$ Daratumumab $16 \mathrm{mg} / \mathrm{kg}$ IV is also approved in combination with bortezomib/melphalan/prednisone or lenalidomide/dexamethasone (in the USA) for the treatment of patients with newly diagnosed MM who are ineligible for autologous stem cell transplantation, and in combination with bortezomib/thalidomide/dexamethasone (in the USA) for patients with newly diagnosed MM who are eligible for autologous stem cell transplantation. ${ }^{8,9}$ In clinical studies of daratumumab, the median durations of the first, second, and subsequent daratumumab IV infusions were 7.0, 4.3, and 3.4 hours (h), respectively. ${ }^{8}$ For the convenience of patients and healthcare providers, the first daratumumab $16 \mathrm{mg} / \mathrm{kg}$ IV dose may be split over 2 days $(8 \mathrm{mg} / \mathrm{kg}$ administered on days 1 and 2 of cycle 1$)$, which is associated with a shorter median infusion duration of $4.2 \mathrm{~h}$ on day 1 of cycle $1 .^{8,10,11}$ In addition to split-dose administration of daratumumab, alternative approaches to reducing the duration of the first daratumumab infusion include administering a low priming dose on day 1 of cycle 1 and the remainder of the $16 \mathrm{mg} / \mathrm{kg}$ IV dose on day 2 , or giving an $8 \mathrm{mg} / \mathrm{kg} \mathrm{IV}$ dose on day 1 of cycle 1 before proceeding with the $16 \mathrm{mg} / \mathrm{kg}$ IV dose from day 8 of cycle 1 onwards. ${ }^{12}$ Furthermore, a shorter, 90-minute (min) infusion for subsequent (day 15 of cycle 1 and beyond) $16 \mathrm{mg} / \mathrm{kg}$ IV administrations of daratumumab was shown to be well tolerated. ${ }^{13}$ Infusion-related reactions (IRR) occur in approximately $50 \%$ of patients treated with IV daratumumab; these reactions are manageable and occur primarily during the first infusion. ${ }^{8}$

Given the infusion time required for IV administration and the incidence of IRR associated with daratumumab, a subcutaneous (SC) delivery method is in development, with the goal of shortening the duration of infusion without compromising the safety or efficacy of the drug. SC administration of daratumumab may be associated with a lower risk of IRR, improved tolerability due to a more gradual systemic absorption of the drug, and greater convenience for both patients and healthcare providers by reducing infusion times.

Recombinant human hyaluronidase $\mathrm{PH} 20$ ( $\mathrm{rHuPH} 20$; ENHANZE $^{\circledast}$ drug delivery technology, Halozyme, Inc., San Diego, CA, USA) depolymerizes hyaluronan in the SC space, allowing rapid administration of large volumes of injected drugs, thus facilitating SC delivery. ${ }^{14} \mathrm{~A}$ mix-anddeliver formulation of daratumumab $(20 \mathrm{mg} / \mathrm{mL})$ and rHuPH20 (DARA-MD), given SC by means of a syringe pump at two dose levels (DARA-MD 1,200 mg and DARA-MD 1,800 mg) over 20 to $30 \mathrm{~min}$, was evaluated in part 1 of PAVO, a phase Ib study in patients with RRMM..$^{15}$ Results from part 1 showed that SC administration of daratumumab is feasible in patients with MM. DARAMD was well tolerated, with low rates of IRR (23\%). The serum concentrations achieved with the $1,800 \mathrm{mg}$ dose of DARA-MD were consistent with those observed for daratumumab $16 \mathrm{mg} / \mathrm{kg}$ IV in patients with RRMM. Moreover, the efficacy of the $1,800 \mathrm{mg}$ dose of DARA$\mathrm{MD}$ was comparable to that of daratumumab $16 \mathrm{mg} / \mathrm{kg}$ IV in a similar population of patients..$^{16}$ At the $1,800 \mathrm{mg}$ dose level of DARA-MD, the overall response rate (ORR) was $42 \%$, and responses were deep and durable, with four $(9 \%)$ patients achieving a stringent complete response. ${ }^{15}$

Based on the results from part 1 of the PAVO study, a concentrated, pre-mixed co-formulation of daratumumab $1,800 \mathrm{mg}(120 \mathrm{mg} / \mathrm{mL})$ and rHuPH20 (DARA SC) with a smaller injection volume $(15 \mathrm{~mL}$ for DARA SC vs. $60 \mathrm{~mL}$ for DARA-MD $1,200 \mathrm{mg}$ and $90 \mathrm{~mL}$ for DARA-MD $1,800 \mathrm{mg}$ ) and shorter injection time was developed, enabling manual SC injection into the abdomen. ${ }^{17}$ This report describes part 2 of $\mathrm{PAVO}$, which investigated the safety, pharmacokinetics (PK), and efficacy of DARA SC in patients with RRMM.

\section{Methods}

\section{Study design and patients}

PAVO (MMY1004) is a phase Ib, open-label, multicenter, dosefinding, proof-of-concept study. Detailed eligibility criteria have already been published. ${ }^{15}$ Briefly, patients were $\geq 18$ years of age, had RRMM and had received two or more prior lines of treatment, including a proteasome inhibitor (PI) and an immunomodulatory drug (IMiD), and were naïve to anti-CD38 therapy. In part 1, DARA-MD was administered by SC infusion over 20 to $30 \mathrm{~min}$ through a syringe pump to determine the recommended dose for part 2. In part 2, a concentrated co-formulation of the selected daratumumab dose $(1,800 \mathrm{mg})$ and $\mathrm{rHuPH} 20$ concentration $(30,000 \mathrm{U}$; in $15 \mathrm{~mL})$ in a single, pre-mixed vial was administered over 3 to $5 \mathrm{~min}$ by manual SC injection (DARA SC) at alternating locations in the periumbilical area of the abdominal wall Treatment was given in 28-day cycles: once weekly during cycles 1 and 2 , every 2 weeks during cycles 3 through 6 , and every 4 weeks thereafter until disease progression or unacceptable toxicity. All patients remained in the hospital for observation for at least $24 \mathrm{~h}$ after the end of the SC injection on day 1 of cycle 1. Inpatient observation after subsequent doses was implemented if deemed necessary based on safety observations.

\section{Endpoints and assessments}

The primary endpoints were daratumumab trough concentration $\left(\mathrm{C}_{\text {trough }}\right)$ at the end of weekly dosing (just prior to administration of the day 1 dose of cycle 3) and safety. Secondary endpoints included ORR and complete response rate.

Blood samples for PK analysis were collected on days 1, 2, 3, 4, 8,15 , and 22 of cycle 1 ; days $1,8,15,22,23$, and 25 of cycle 2 ; day 1 of cycles $3,4,6$, and 8 ; and 4 and 8 weeks after the final dose of study medication. Blood samples collected prior to dosing on days 1 and 15 of cycle 1 , day 22 of cycle 2 , and day 1 of cycle 4 as well as 4 and 8 weeks after the end of treatment were assessed for anti-daratumumab antibodies and anti-rHuPH20 antibodies.

Safety assessments included adverse-event monitoring, physical examinations, electrocardiograms, injection-site evaluations, clinical laboratory parameters, vital sign measurements, and Eastern Cooperative Oncology Group Performance Status. Adverse events were assessed using National Cancer Institute Common Terminology Criteria for Adverse Events Version 4.03. ${ }^{18}$

Responses were assessed according to International Myeloma Working Group consensus recommendations ${ }^{19,20}$ at the beginning 
of each cycle. Disease evaluations were performed by a central laboratory until disease progression or end of treatment.

\section{Statistical analyses}

The primary endpoint of $\mathrm{C}_{\text {trough }}$ at the end of weekly dosing (prior to the day 1 dose of cycle 3) was evaluated in patients who received all eight weekly doses of DARA SC and provided a predose PK sample on day 1 of cycle 3 (PK-evaluable population). All other PK analyses were performed on the PK-analysis population, which included patients who received at least one dose of study drug and provided one or more post-infusion PK samples. The safety population included all patients who received at least one dose of study drug.

The study protocol was approved by the clinical study sites' institutional review boards or ethics committees. Additional details on the study design, statistical analyses, and study supervision are provided in the Online Supplementary Appendix.

\section{Results}

\section{Patients and treatment}

At the clinical cutoff date of December 14, 2018, 25 patients had been enrolled in part 2 of the study and had received DARA SC 1,800 mg. The patients' median age was 68 (range, $51-85$ ) years, and $24 \%$ of patients were $\geq 75$ years of age (Table 1 ). The median number of prior lines of therapy was three (range, $2-9$ ); $96 \%$ and $92 \%$ of patients had received prior treatment with bortezomib and lenalidomide, respectively. Fifty-six percent of patients were refractory to both a PI and an IMiD, and $76 \%$ of patients were refractory to the last line of therapy. Of the 16 patients with available cytogenetic data, four $(25 \%)$ had a high-risk cytogenetic abnormality at baseline: two had del17p, one had $t(4 ; 14)$, and one had $t(14 ; 16)$.

Of the 25 patients treated with DARA SC during part 2 of the study, 19 (76\%) discontinued treatment: 17 (68\%) due to progressive disease and two (8\%) due to physicians' decision (both had unconfirmed progressive disease). At the clinical cutoff date, the median duration of follow-up was 14.2 (range, 2.4-18.5) months.

\section{Pharmacokinetics and immunogenicity}

PK results for $C_{\text {trough }}$ on day 1 of cycle 3 are presented for the PK-evaluable population $(\mathrm{n}=22)$; all other PK data are presented based on the PK-analysis population $(n=25)$. The linear mean daratumumab serum concentration profiles after cycle 1 (first dose) and after the last weekly dose (eighth dose) are shown in Figure 1. Daratumumab serum concentrations after SC administration were sustained from $48 \mathrm{~h}$ post-dose onwards until the end of the dosing interval (Figure 1A). SC administration of daratumumab was followed by slow systemic absorption as opposed to the immediate systemic availability following IV administration (Figure 1A). However, the maximum $\mathrm{C}_{\text {trough }}$ was similar or higher following DARA SC 1,800 $\mathrm{mg}$ than after the $16 \mathrm{mg} / \mathrm{kg}$ IV dosing of daratumumab (Figure $1 \mathrm{~B}$ and Table 2).

Simulation of mean concentration-time profiles of daratumumab following SC and IV dosing revealed that the $\mathrm{C}_{\text {trough }}$ following DARA SC 1,800 mg dosing remained higher than after $16 \mathrm{mg} / \mathrm{kg}$ IV dosing throughout the dosing regimen (Figure 2). For SC dosing, the mean maximum concentration $\left(\mathrm{C}_{\max }\right)$ was lower during early weekly dosing but was higher at the end of weekly dosing and during every-2-week dosing (Figure 2). After reaching every-4week dosing, the $\mathrm{C}_{\max }$ for DARA SC 1,800 mg was similar to that for the $16 \mathrm{mg} / \mathrm{kg}$ IV dosing of daratumumab overall (Figure 2). The mean and median $\mathrm{C}_{\text {trough }}$ values for end of weekly dosing (day 1 of cycle 3 ) in PAVO part 2, as well as in patients receiving daratumumab $16 \mathrm{mg} / \mathrm{kg}$ IV as monotherapy in the GEN501 part 2 and SIRIUS studies, are summarized in Table 2.

Of the 25 patients, one (4\%) patient was positive for anti-daratumumab antibodies; the antibodies were neutralizing and transient (detected only at week 4 after treatment). This patient experienced no IRR and had a best response of stable disease. One (4\%) patient was positive for anti-rHuPH20 antibodies at baseline, and four (16\%) patients were positive for anti-rHuPH20 antibodies during treatment; all the antibodies were non-neutralizing. Among the four patients who were positive for anti-

Table 1. Baseline demographics and clinical characteristics of the all-treated population.

\begin{tabular}{|c|c|}
\hline Characteristic & $\begin{array}{c}\text { DARA SC 1,800 mg } \\
(n=25)\end{array}$ \\
\hline $\begin{array}{l}\text { Age, years } \\
\text { Median (range) } \\
\geq 75, \mathrm{n}(\%)\end{array}$ & $\begin{array}{l}68(51-85) \\
6(24.0)\end{array}$ \\
\hline Median (range) weight, kg & $70.9(52.0-104.8)$ \\
\hline $\begin{array}{l}\text { Baseline ECOG Performance Status, n (\%) } \\
0 \\
1 \\
2\end{array}$ & $\begin{array}{c}11(44.0) \\
13(52.0) \\
1(4.0)\end{array}$ \\
\hline $\begin{array}{l}\text { ISS stage, } \mathrm{n}^{\mathrm{a}} \\
\text { I, n (\%) } \\
\text { II, n (\%) } \\
\text { III, n (\%) }\end{array}$ & $\begin{array}{l}24 \\
13(54.2) \\
5(20.8) \\
6(25.0)\end{array}$ \\
\hline Median (range) time from diagnosis, years & $5.9(2.1-12.8)$ \\
\hline $\begin{array}{l}\text { Type of myeloma, } \mathrm{n} \\
\operatorname{IgG}, \mathrm{n}(\%)\end{array}$ & $\begin{array}{c}24 \\
13(54.2)\end{array}$ \\
\hline $\begin{array}{l}\text { Cytogenetic risk, } \mathrm{n}^{\mathrm{b}} \\
\text { Standard risk, } \mathrm{n}(\%) \\
\text { High risk, } \mathrm{n}(\%) \\
\mathrm{t}(4 ; 14) \\
\mathrm{t}(14 ; 16) \\
\text { dell7p }\end{array}$ & $\begin{array}{l}16 \\
12(75.0) \\
4(25.0)^{c} \\
1(6.3) \\
1(6.3) \\
2(12.5)\end{array}$ \\
\hline $\begin{array}{l}\text { Prior lines of therapy, n (\%) } \\
\text { Median (range) } \\
\leq 3 \\
>3\end{array}$ & $\begin{array}{l}3(2-9) \\
17(68.0) \\
8(32.0)\end{array}$ \\
\hline Prior ASCT, n (\%) & $17(68.0)$ \\
\hline $\begin{array}{l}\text { Prior PI, } \mathrm{n}(\%) \\
\text { Bortezomib }\end{array}$ & $\begin{array}{l}25(100) \\
24(96.0)\end{array}$ \\
\hline $\begin{array}{l}\text { Prior IMiD, n (\%) } \\
\text { Lenalidomide }\end{array}$ & $\begin{array}{l}25(100) \\
23(92.0)\end{array}$ \\
\hline $\begin{array}{l}\text { Refractory to, } \mathrm{n}(\%) \\
\text { Bortezomib } \\
\text { Lenalidomide } \\
\text { Both PI and IMiD } \\
\text { Last line of therapy }\end{array}$ & $\begin{array}{l}16(64.0) \\
14(56.0) \\
14(56.0) \\
19(76.0)\end{array}$ \\
\hline \multicolumn{2}{|c|}{$\begin{array}{l}\text { DARA: daratumumab; SC: subcutaneous; ECOG: Eastern Cooperative Oncology Group; ISS } \\
\text { International Staging System; ASCT: autologous stem cell transplantation; PI: proteasome } \\
\text { inhibitor; IMiD: immunomodulatory drug. }{ }^{2} \text { ISS stage is derived based on the combination of } \\
\text { serum } \beta_{2} \text {-microglobulin and albumin. }{ }^{\circ} \text { Assessed by fluorescence in situ hybridization or kary } \\
\text { otyping. }{ }^{-} \text {Consists of two patients with del17p, one patient with } \mathrm{t}(4 ; 14) \text {, and one patient with } \\
\mathrm{t}(14 ; 16) \text {. }\end{array}$} \\
\hline
\end{tabular}


A

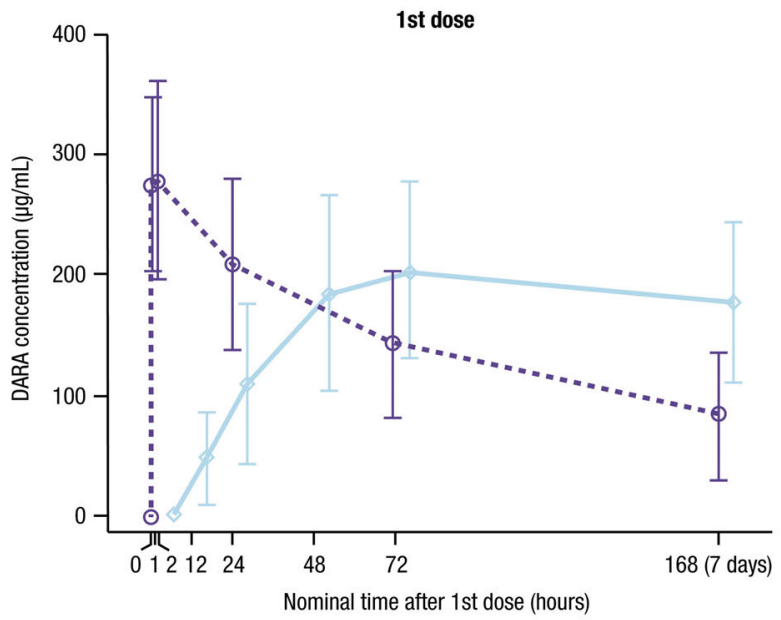

B

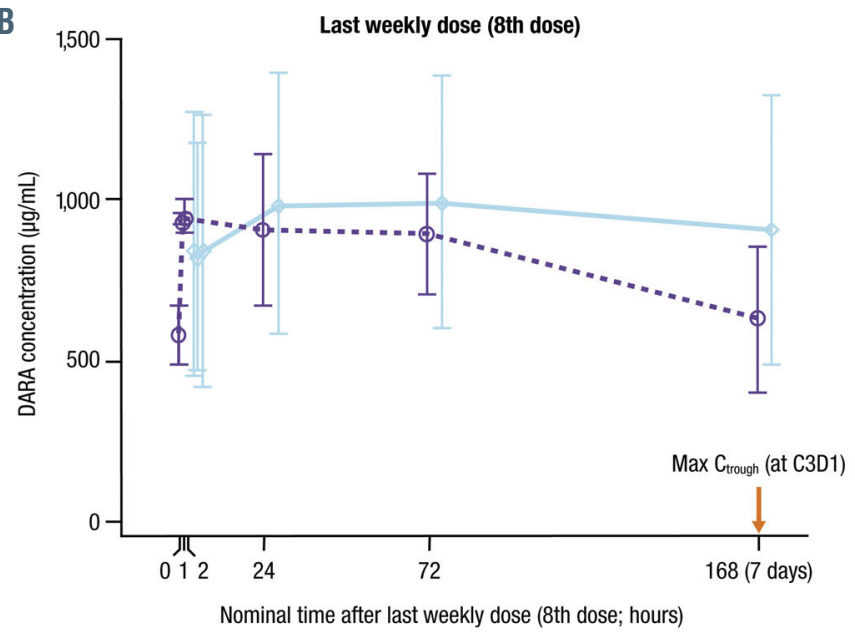

$\rightarrow 16 \mathrm{mg} / \mathrm{kg} \mathrm{IV} \mathrm{V}^{\mathrm{a}} \diamond 1,800 \mathrm{mg} \mathrm{SC}$

Figure 1. Serum concentrations of daratumumab over time. The mean ( \pm standard deviation) serum concentrations of daratumumab over time (A) after the first dose and (B) after the last weekly dose (eighth dose). DARA: daratumumab; $\mathrm{C}_{\text {trough }}$ : trough concentration; C3D1: day 1 of cycle 3; IV: intravenous; SC: subcutaneous. ${ }^{a}$ From the GEN501 study. ${ }^{16}$

Table 2. $C_{\text {trough }}$ for end of weekly daratumumab dosing (day 1 of cycle 3) for DARA SC $1,800 \mathrm{mg}$ and historical data for daratumumab $16 \mathrm{mg} / \mathrm{kg}$ IV.

\begin{tabular}{|c|c|c|c|c|c|}
\hline \multirow[b]{2}{*}{ Study } & \multirow[b]{2}{*}{ Dose/route } & \multirow[b]{2}{*}{ n } & \multicolumn{3}{|c|}{ Daratumumab cycle 3 , day $1 \mathrm{C}_{\text {trough }}(\mu \mathrm{g} / \mathrm{mL})$} \\
\hline & & & Mean & Median & CV\% \\
\hline $\mathrm{PAVO}^{\mathrm{a}}$ & $1,800 \mathrm{mg} \mathrm{SC}$ & 22 & 932 & 860 & $42 \%$ \\
\hline GEN501 part 2 & $16 \mathrm{mg} / \mathrm{kg} \mathrm{IV}$ & 27 & 617 & 714 & $51 \%$ \\
\hline SIRIUS & $16 \mathrm{mg} / \mathrm{kg} \mathrm{IV}$ & 73 & $573^{\mathrm{b}}$ & 560 & $58 \%$ \\
\hline
\end{tabular}

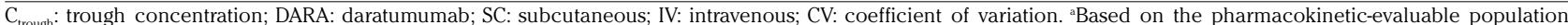
${ }^{\mathrm{b}}$ As reported by Clemens et al. ${ }^{22}$

rHuPH20 antibodies during treatment, one patient experienced a mild IRR on day 1 of cycle 1, and best responses were partial response (1 patient), stable disease ( 2 patients), and progressive disease (1 patient).

\section{Safety}

The adverse event profile of DARA SC was consistent with the known safety and tolerability profile of IV daratumumab. The most frequently reported treatment-emergent adverse events (TEAE) were lymphopenia (32\%), arthralgia (28\%), back pain (28\%), and thrombocytopenia, diarrhea, and nasopharyngitis (24\% each) (Table 3$)$. The most common grade $3 / 4$ TEAE was lymphopenia (20\%) (Table 3 ). No treatment discontinuations due to TEAE were observed. Serious TEAE were reported in six $(24 \%)$ patients who received DARA SC but were not considered related to the study drug. One patient died during part 2 of the study due to disease progression in the context of MM-related amyloid light chain (AL) amyloidosis (clinically significant cardiac involvement was not present); this death was not considered related to DARA SC. A secondary primary malignancy was observed in one patient (metastatic adenocarcinoma of prostate). There were no reports of tumor lysis syndrome or intravascular hemolysis.

The incidence and severity of IRR was low with DARA SC $1,800 \mathrm{mg}$. Among the 25 patients who received DARA SC $1,800 \mathrm{mg}$, four $(16 \%)$ reported IRR, the majority of which occurred on day 1 of cycle 1 . The median time to onset of an IRR was 70 (range, 9-80) min. Patient 1 experienced grade 3 hypertension, grade 2 chills, and grade 2 dys- pnea. Patient 2 experienced grade 1 allergic rhinitis, patient 3 experienced grade 1 sneezing, and patient 4 experienced grade 3 hypertension. Patients 1, 2, and 3 experienced their IRR following the first injection (day 1 of cycle 1 ), whereas patient 4 experienced the IRR following the ninth injection (day 1 of cycle 3 ). The IRR of grade 3 hypertension were reversible, and both occurred in patients with a medical history of hypertension. There were no grade 4 IRR and no discontinuations due to IRR.

Injection-site TEAE were observed in three (12\%) patients; these included injection-site induration, injectionsite discoloration, erythema, and hematoma ( $n=1$ each; all grade 1 severity). Erythema and induration were objectively measured for all injections, regardless of attribution as a TEAE. Measurable erythema (24\%) and measurable induration $(4 \%)$ at the injection site resolved within $1 \mathrm{~h}$.

\section{Efficacy}

At a median follow-up of 14.2 months, the ORR with DARA SC $1,800 \mathrm{mg}$ was $52 \%$, which included a complete response in one (4\%) patient, very good partial responses in seven $(28 \%)$ patients, and partial responses in five $(20 \%)$ patients (Figure 3A). The median time to best response was 1.02 (range, 1.0-12.1) months, and the median duration of response was 15.7 (range, 4.6-not estimable) months. Among 13 responders, three responses deepened over time (Figure 3B); one patient with an initial partial response at month 2 went on to achieve a complete response at month 12 , and two patients who achieved initial partial responses at month 1 went on to achieve very good partial responses 


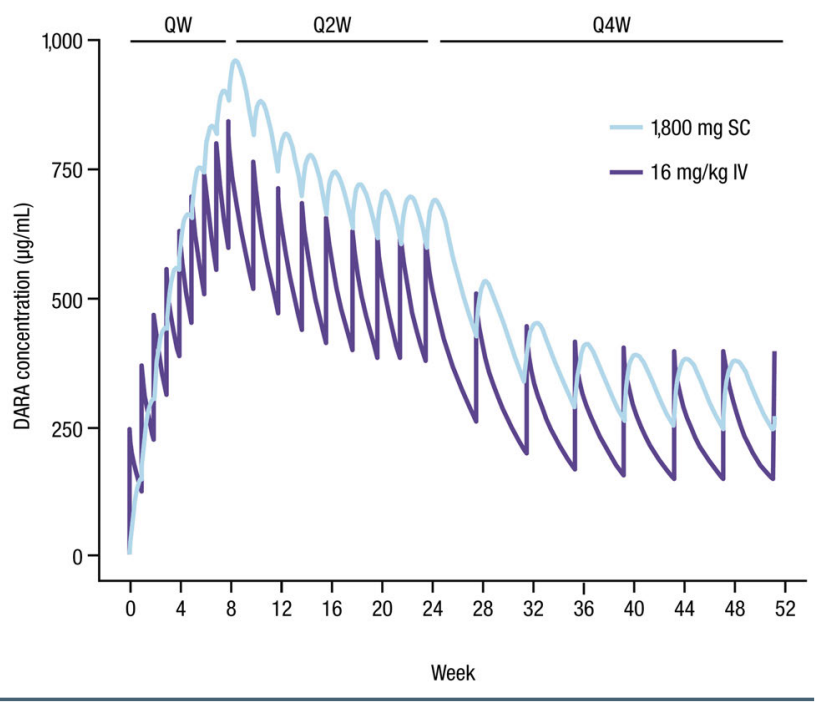

Figure 2. Simulation of mean concentration-time profiles of daratumumab following subcutaneous and intravenous dosing. ${ }^{\mathrm{a}, \mathrm{b}} \mathrm{QW}$ : weekly; Q2W: every 2 weeks; O4W: every 4 weeks; SC: subcutaneous; IV: intravenous; DARA: daratumumab. ${ }^{a}$ The dosing schedule is once weekly in cycles 1 and 2 , every 2 weeks in cycles 3 through 6 , and every 4 weeks thereafter. 'Simulations were conducted based on a population pharmacokinetic (PK) model developed for daratumumab following SC and IV administration using scheduled dosing and estimated individual PK parameters. Serum concentration-time data (from the PAVO, GEN501, and SIRIUS studies) were used for the population PK model development using a nonlinear mixed-effects modeling (NONMEM ${ }^{\oplus}$, version 7.2) approach with the first-order conditional estimation with interaction method. The daratumumab SC model was based on a previous population PK model for daratumumab IV ${ }^{21}$ except for the absorption, which was described by a first-order process and a relative bioavailability parameter.

at month 2. At the clinical cutoff, the median progressionfree survival was 12.0 (range, 5.6-16.6) months among alltreated patients and 11.7 (range, 2.8-13.8) months among patients refractory to both PI and IMiD.

\section{Discussion}

PAVO is the first clinical study to evaluate the safety, PK, and efficacy of SC administration of daratumumab in combination with rHuPH20. The results from this study demonstrate that daratumumab co-formulated with rHuPH20 is well tolerated, has an acceptable PK profile, and achieves deep and durable responses in patients with RRMM. In part 1 of the study, dose escalation of a first-generation mix-and-deliver formulation of daratumumab and rHuPH20 (DARA-MD) showed that daratumumab SC administration is feasible in patients with RRMM. ${ }^{15}$ DARA$\mathrm{MD} 1,800 \mathrm{mg}$ had a $\mathrm{PK}$ profile and produced clinical responses that were consistent with those of IV infusion of daratumumab $16 \mathrm{mg} / \mathrm{kg}$ and was well tolerated, with a low rate of IRR. Based on the promising safety and efficacy observed with DARA-MD during part 1 of PAVO, a premixed co-formulation of daratumumab 1,800 $\mathrm{mg}$ with rHuPH20 (DARA SC), which has a smaller injection volume and is administered over 3 to $5 \mathrm{~min}$, was investigated in part 2 of the study.

Population PK and exposure-response analyses of daratumumab IV monotherapy in patients with MM revealed that the daratumumab maximum $\mathrm{C}_{\text {trough }}$ is strongly related to ORR. ${ }^{21}$ In these studies, the maximum $\mathrm{C}_{\text {trough }}$ occurred prior to daratumumab dosing on day 1 of cycle 3 with daratumumab monotherapy administered with the same dosing
Table 3. Treatment-emergent adverse events.

\begin{tabular}{|c|c|c|}
\hline \multirow[t]{2}{*}{ TEAE, n (\%) } & \multicolumn{2}{|c|}{ DARA SC 1,800 mg (n=25) } \\
\hline & $\begin{array}{l}\text { All grades } \\
>10 \%\end{array}$ & $\begin{array}{c}\text { Grade } 3 \text { or } 4 \\
>1 \text { patient }\end{array}$ \\
\hline \multicolumn{3}{|l|}{ Hematologic } \\
\hline Lymphopenia & $8(32.0)$ & $5(20.0)$ \\
\hline Thrombocytopenia & $6(24.0)$ & $2(8.0)$ \\
\hline Anemia & $4(16.0)$ & $1(4.0)$ \\
\hline Leukopenia & $3(12.0)$ & $1(4.0)$ \\
\hline Neutropenia & $2(8.0)$ & $2(8.0)$ \\
\hline \multicolumn{3}{|l|}{ Non-hematologic } \\
\hline Arthralgia & $7(28.0)$ & 0 \\
\hline Back pain & $7(28.0)$ & 0 \\
\hline Diarrhea & $6(24.0)$ & $1(4.0)$ \\
\hline Nasopharyngitis & $6(24.0)$ & 0 \\
\hline Hypertension & $5(20.0)$ & $2(8.0)$ \\
\hline Fatigue & $5(20.0)$ & $1(4.0)$ \\
\hline Asthenia & $5(20.0)$ & $1(4.0)$ \\
\hline Insomnia & $5(20.0)$ & $1(4.0)$ \\
\hline Nausea & $5(20.0)$ & 0 \\
\hline Headache & $5(20.0)$ & 0 \\
\hline Upper RTI & $5(20.0)$ & 0 \\
\hline Pyrexia & $5(20.0)$ & 0 \\
\hline Cough & $5(20.0)$ & 0 \\
\hline Vomiting & $4(16.0)$ & 0 \\
\hline Constipation & $4(16.0)$ & 0 \\
\hline Musculoskeletal pain & $4(16.0)$ & 0 \\
\hline Oropharyngeal pain & $4(16.0)$ & 0 \\
\hline Bone pain & $3(12.0)$ & $1(4.0)$ \\
\hline Chills & $3(12.0)$ & 0 \\
\hline Peripheral edema & $3(12.0)$ & 0 \\
\hline Musculoskeletal chest pain & $3(12.0)$ & 0 \\
\hline Musculoskeletal discomfort & $3(12.0)$ & 0 \\
\hline Dyspnea & $3(12.0)$ & 0 \\
\hline
\end{tabular}

TEAE: treatment-emergent adverse event; DARA: daratumumab; SC: subcutaneous; RTI: respiratory tract infection.

schedule used in the PAVO study. ${ }^{22}$ Based on these findings, $\mathrm{C}_{\text {trough }}$ at the end of weekly dosing (just prior to the dose on day 1 of cycle 3 ) was selected as the primary PK endpoint for the PAVO study. DARA SC 1,800 mg achieved similar or greater maximum $\mathrm{C}_{\text {trough }}$ values compared with those of standard IV dosing $(16 \mathrm{mg} / \mathrm{kg})$ at day 1 of cycle 3 (i.e., at the end of weekly dosing). The mean $\mathrm{C}_{\text {trough }}$ at day 1 of cycle 3 (pre-dose) was $932 \mu \mathrm{g} / \mathrm{mL}$ after eight weekly doses of DARA SC 1,800 mg compared with $617 \mu \mathrm{g} / \mathrm{mL}$ (GEN501 part 2) and $573 \mu \mathrm{g} / \mathrm{mL}$ (SIRIUS) after weekly dosing with IV daratumumab. ${ }^{16,22}$ Daratumumab serum concentrations were sustained from $48 \mathrm{~h}$ post-dose onwards until the end of the dosing interval. Although DARA SC is administered at a fixed dose, while IV daratumumab is administered by weight-based dosing, interpatient $\mathrm{PK}$ variability of the two dosing methods was comparable. ${ }^{23,24}$ Due to the small sample size in part 2 of PAVO, the relationship between the PK of DARA SC and patients' body weight could not be assessed. However, in a body-weight-based subgroup analysis of the phase III COLUMBA study (NCT03277105), DARA SC achieved adequate exposure that was consistent with that of IV daratumumab, regardless of body weight. ${ }^{25}$ In patients weighing $\leq 65 \mathrm{~kg}$, the higher mean maximum $\mathrm{C}_{\text {trough }}$ observed with DARA SC versus IV daratumumab did not have a clinically relevant effect on safety, and the ORR in all subgroups were consistent with the overall population. These results suggest that dose individualization of DARA SC on the basis of body weight is not necessary. 


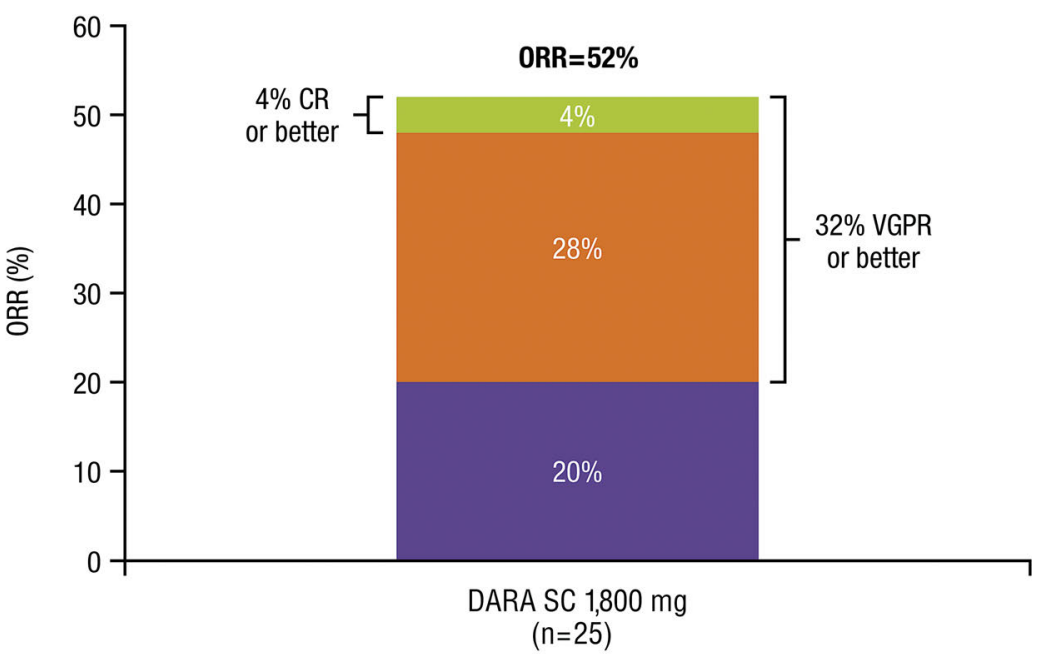
cutaneous; ORR: overall response rate; CR: complete response; VGPR: very good partial response; PR: partial response.

B

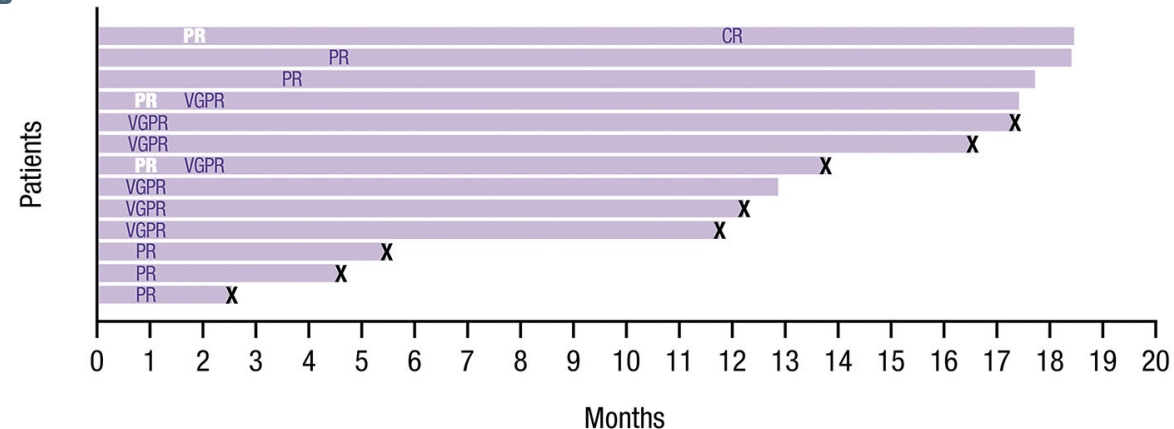

There was a low incidence of anti-daratumumab antibodies with DARA SC, indicating a low risk of daratumum$\mathrm{ab}$ immunogenicity following SC administration. Few patients $(16 \%)$ were positive for treatment-emergent or treatment-induced anti-rHuPH20 antibodies; this rate is consistent with the immunogenicity rates reported for other $\mathrm{rHuPH} 20$-containing treatments (SC rituximab [9\%], trastuzumab [20\%], and immune globulin infusion HYQVIA [18\%]). ${ }^{26-28}$

The safety profile of DARA SC was consistent with the known profile of IV daratumumab; however, the frequency of IRR was lower with the SC administration than with the IV administration. The IRR rate with DARA SC was $16 \%$, while IRR rates for IV administration of daratumumab range from $45 \%$ to $56 \%$ in monotherapy and combination regimens for RRMM. ${ }^{29-34}$ The majority of IRR occurred on day 1 of cycle 1 . Only two patients experienced grade 3 IRR, and no cases of grade 4 IRR were reported. There were no discontinuations due to IRR or other TEAE. DARA SC injections in the periumbilical area were well tolerated; few injection-site TEAE were observed, and all objectively measured cases of erythema and induration were fully reversible and resolved within $1 \mathrm{~h}$ after the end of the injection.

Durable clinical responses were observed with DARA SC during this study, with a median duration of response of 15.7 months. Over time, the ORR improved, increasing from $44 \%$ to $52 \%$ after a median follow-up of $4.6^{17}$ versus 14.2 months, respectively, and responses deepened, with one patient achieving a complete response with longer follow-up. Despite the sample size of 25 patients, the median progression-free survival was 12.0 months among all-treat- ed patients and 11.7 months among patients refractory to both PI and IMiD. An ORR of $52 \%$ is noteworthy in this population, with patients having received a median of three (range, 2-9) prior lines of therapy and $56 \%$ of patients being refractory to both $\mathrm{PI}$ and IMiD and $76 \%$ of patients being refractory to their last line of therapy. Clinical response rates achieved with DARA SC 1,800 mg were comparable to those achieved with daratumumab $16 \mathrm{mg} / \mathrm{kg} \mathrm{IV.}$. $^{16,35}$ In a pooled analysis of 148 patients with RRMM who received daratumumab $16 \mathrm{mg} / \mathrm{kg}$ IV in the monotherapy studies GEN501 and SIRIUS, the ORR was 31\%, with 14\% of patients achieving a very good partial response or better and $5 \%$ achieving a complete response or better. ${ }^{31}$ After a median follow-up of 20.7 months, the median progressionfree survival was 4.0 months. ${ }^{31}$ The pooled patient population received a median of five (range, 2-14) prior lines of therapy, $87 \%$ were considered double refractory to a PI and an $\mathrm{IMiD}$, and $91 \%$ were considered refractory to the last line of therapy.

In part 1 of PAVO, DARA-MD was administered by means of an infusion pump over a period of approximately 20 to $30 \mathrm{~min}^{15}$ Compared with DARA-MD, the DARA SC formulation contains a higher concentration of daratumumab in a smaller injection volume $(15 \mathrm{~mL})$, thus enabling drug delivery through a single SC injection with a handheld syringe and needle by manual push into the abdominal wall over 3 to $5 \mathrm{~min}$. As the median durations of the first, second, and subsequent daratumumab IV infusions in clinical studies were 7.0, 4.3, and 3.4 hours, respectively, ${ }^{8}$ DARA SC substantially shortens the duration of administration, thereby reducing the treatment 
burden for patients and healthcare providers.

Findings from PAVO on the safety, PK, and efficacy profile of DARA SC informed four ongoing phase III studies of DARA SC 1,800 mg. ${ }^{36-39}$ In the randomized, multicenter, open-label, non-inferiority study, COLUMBA, DARA SC demonstrated non-inferiority to DARA $16 \mathrm{mg} / \mathrm{kg}$ IV in terms of ORR and maximum $\mathrm{C}_{\text {trough }}$ on day 1 of cycle 3 , with a similar safety profile and a significantly reduced IRR rate, in patients with RRMM. ${ }^{36}$ Patient-reported outcome data from COLUMBA indicated that patients receiving DARA SC were more satisfied with their cancer therapy and had more positive perceptions of their treatment than patients receiving IV daratumumab. ${ }^{40}$ The efficacy and safety of DARA SC are also being investigated in combination with standard-of-care regimens in the phase II PLEIADES study, and results from this study demonstrate the feasibility of administering DARA SC in combination with standard-of-care regimens containing SC bortezomib. In PLEIADES, DARA SC in combination with SC bortezomib plus lenalidomide/dexamethasone and with SC bortezomib plus melphalan/prednisone in patients with newly diagnosed $\mathrm{MM}$ demonstrated comparable clinical activity and safety to the corresponding IV daratumumab regimens, with lower rates of IRR and shorter durations of administration. ${ }^{41}$ Although the dose of DARA SC on day 1 of cycle 1 should not be given at home, at-home administration of subsequent doses of DARA SC by a healthcare provider is a possibility for future consideration; however, additional safety follow-up is needed, particularly to further confirm that the risk of severe IRR with administration beyond the first day of cycle 1 is very low or absent.

Taken together, these findings show that DARA SC is well tolerated, with a low rate of IRR and a shortened administration time. No new safety concerns were identified, and the overall adverse event profile with SC administration was comparable to that reported with IV administration of daratumumab at the approved dose level $(16 \mathrm{mg} / \mathrm{kg}){ }^{16,35}$ DARA SC achieved maximum $\mathrm{C}_{\text {trough }}$ values that were similar to or greater than the maximum $\mathrm{C}_{\text {trough }}$ observed for the approved $16 \mathrm{mg} / \mathrm{kg}$ IV dose following the same dosing schedule. DARA SC demonstrated robust clinical efficacy, producing deep and durable responses with a rapid onset. Collectively, these data indicate that, compared to the current IV formulation, DARA SC reduces administration time as well as IRR rates without compromising safety or efficacy. Based on these results, ongoing studies are actively investigating DARA SC in MM and other disease states.

\section{Disclosures}

JS-M has acted as a consultant for Amgen, Bristol Myers Squibb, Celgene, Janssen, Merck, Novartis, Takeda, Sanofi, and Roche. SZU has acted as a consultant for AbbVie, GlaxoSmithKline, Celgene, Amgen/Onyx, Takeda/Millennium, Sanofi, Seattle Genetics, Skyline, Merck, and Janssen; has received research funding from Celgene, Amgen/Onyx, Takeda/Millennium, Sanofi, Seattle Genetics, Skyline, Merck, Janssen, Array BioPharma, and
Pharmacyclics; has served on speakers' bureaus for Celgene, Amgen, Janssen, Sanofi, and Takeda; and has received travel expenses from Janssen, Celgene, Amgen, and Takeda. M-VM has received honoraria from and has acted as a consultant for Celgene, Janssen, Takeda, and Amgen. NWCJvdD has received research support from Janssen Pharmaceuticals, Amgen, Celgene, Novartis, and Bristol Myers Squibb and has served on advisory boards for Janssen Pharmaceuticals, Amgen, Celgene, Bristol Myers Squibb, Novartis, Bayer, Takeda, and Servier. JLK has acted as a consultant or served in an advisory role for Janssen, Takeda, Celgene, Bristol Myers Squibb, Karyopharm Therapeutics, TG Therapeutics, Sanofi, Amgen, and Tecnofarma; has received research funding (institutional) from Merck, Celgene, Janssen, Sutro Biopharma, Fortis Therapeutics, Amgen, AbbVie/Genentech, and Bristol Myers Squibb; and has received travel and accommodation expenses from Janssen, Celgene, Bristol Myers Squibb, Sanofi, Amgen, and Takeda. PM has acted as a consultant for and received honoraria from Celgene, Takeda, and Janssen. AO has acted as a consultant for and received honoraria from Amgen, Takeda, and Janssen and has served on speakers' bureaus for Amgen, Celgene, and Janssen. $T P$ has received research support from Janssen Pharmaceuticals and served on advisory boards for Janssen Pharmaceuticals, Celgene, Takeda, and Behring. $L B$ has acted as a consultant for and received honoraria from Takeda, Celgene, Janssen, and Amgen and has received travel expenses from Janssen, Celgene, and Amgen. $K L$, $P H, T M, P L C, M L$, and $A F$ are employees of Janssen. PH, TM, PLC, ML, and AF hold stock in Johnson \& Johnson. AC has acted as a consultant for Amgen, Array BioPharma, Celgene, Janssen, Millennium, Takeda, and Novartis and has received research funding from Amgen, Array BioPharma, Celgene, Janssen, Millennium, Takeda, Novartis, and Pharmacyclics. HN has no conflicts of interest to report.

\section{Contributions}

All authors developed the manuscript, provided final submission approval, and confirmed that the protocol was followed and that the data were accurate and complete.

\section{Acknowledgments}

This study was sponsored by Janssen Research \& Development, LLC. The authors would like to thank the patients who participated in this study and their families, as well as the study co-investigators, research nurses, and coordinators at each of the clinical sites. Medical writing and editorial support were provided by Kristin Runkle, PhD, of MedErgy, and were funded by Janssen Global Services, LLC.

\section{Funding}

The study was registered at ClinicalTrials.gov (NCT02519452) and was sponsored by Janssen Research \& Development, LLC. Medical writing and editorial support were funded by Janssen Global Services, LLC. The data-sharing policy of Janssen Pharmaceutical Companies of Johnson \& Johnson is available at https://www.janssen.com/clinical-trials/transparency. As noted on this site, requests for access to the study data can be submitted through the Yale Open Data Access (YODA) Project site at http://yoda.yale.edu.

\section{References}

1. de Weers M, Tai YT, van der Veer MS, et al. Daratumumab, a novel therapeutic human CD38 monoclonal antibody, induces killing of multiple myeloma and other hematologi- cal tumors. J Immunol. 2011;186(3):18401848.

2. Lammerts van Bueren J, Jakobs D, Kaldenhoven N, et al. Direct in vitro comparison of daratumumab with surrogate analogs of CD38 antibodies MOR03087, SAR650984 and Ab79. Blood. 2014;124(21):3474.

3. Overdijk MB, Verploegen S, Bogels M, et al. Antibody-mediated phagocytosis contributes to the anti-tumor activity of the therapeutic antibody daratumumab in lymphoma and multiple myeloma. MAbs. 
2015;7(2):311-321.

4. Overdijk MB, Jansen JH, Nederend $M$, et al. The therapeutic CD38 monoclonal antibody daratumumab induces programmed cell death via Fcy receptor-mediated cross-linking. J Immunol. 2016;197(3):807-813.

5. Krejcik J, Casneuf T, Nijhof IS, et al. Daratumumab depletes CD38+ immuneregulatory cells, promotes T-cell expansion, and skews T-cell repertoire in multiple myeloma. Blood. 2016;128(3):384-394.

6. Chiu C, Casneuf T, Axel A, et al. Daratumumab in combination with lenalidomide plus dexamethasone induces clonality increase and T-cell expansion: results from a phase 3 randomized study (POLLUX). Presented at: the 58th American Society of Hematology (ASH) Annual Meeting \& Exposition; December 3-6, 2016; San Diego, CA. Abstract 4531.

7. Adams HC III, Stevenaert F, Krejcik J, et al. High-parameter mass cytometry evaluation of relapsed/refractory multiple myeloma patients treated with daratumumab demonstrates immune modulation as a novel mechanism of action. Cytometry A. 2019;95(3): 279-289.

8. DARZALEX ${ }^{\circledast}$ (daratumumab) injection, for intravenous use [package insert]. Horsham, PA: Janssen Biotech, Inc; 2019.

9. European Medicines Agency. Summary of opinion (post authorisation). DARZALEX (daratumumab). https://www.ema.europa. eu/en/documents/smop/chmp-post-authorisation-summary-positive-opinion-darzalexii-11_en.pdf. Published July 2018. Accessed August 2019.

10. Jakubowiak A, Chari A, Lonial S, et al. Daratumumab (DARA) in combination with carfilzomib, lenalidomide, and dexamethasone (KRd) in patients (pts) with newly diagnosed multiple myeloma (MMY1001): an open-label, phase $1 \mathrm{~b}$ study. Presented at: the Annual Meeting of the American Society of Clinical Oncology (ASCO); June 2-6, 2017; Chicago, IL. Abstract 8000.

11. Chari A, Martinez-Lopez J, Mateos MV, et al. Daratumumab in combination with carfilzomib and dexamethasone (D-Kd) in lenalidomide-refractory patients with relapsed multiple myeloma: subgroup analysis of MMY1001. Presented at: the Annual Meeting of the American Society of Clinical Oncology (ASCO); June 1-5, 2018; Chicago, IL. Abstract 8002 .

12. Scheid C, Munder M, Salwender H, Engelhardt $M$. Infusion of daratumumab in combination therapies - practical information for the outpatient area. Dtsch Med Wochenschr. 2018;143(16):1201-1206.

13. Barr H, Dempsey J, Waller A, et al. Ninetyminute daratumumab infusion is safe in multiple myeloma. Leukemia. 2018;32(11):24952518.

14. Halozyme Therapeutics. Mechanism of action for Hylenex recombinant (hyaluronidase human injection). www.hylenex.com/mechanism-of-action. Accessed August 2019.

15. Usmani SZ, Nahi H, Mateos MV, et al. Subcutaneous delivery of daratumumab in relapsed or refractory multiple myeloma. Blood. 2019;134(8):668-677.

16. Lokhorst HM, Plesner T, Laubach JP, et al. Targeting CD38 with daratumumab monotherapy in multiple myeloma. $\mathrm{N}$ Engl J Med. 2015;373(13):1207-1219.

17. Chari A, Nahi H, Mateos MV, et al.
Subcutaneous delivery of daratumumab in patients (pts) with relapsed or refractory multiple myeloma (RRMM): PAVO, an open-label, multicenter, dose escalation phase $1 \mathrm{~b}$ study. Presented at: the 59th American Society of Hematology (ASH) Annual Meeting \& Exposition; December 912, 2017; Atlanta, GA. Abstract 838.

18. US Department of Health and Human Services, National Institutes of Health, National Cancer Institute. Common terminology criteria for adverse events (CTCAE): Version 4.03. https://evs.nci.nih.gov/ftp1/ CTCAE/CTCAE_4.03/CTCAE_4.03_201006-14_QuickReference_5x7.pdf. Accessed August 2019.

19. Durie BGM, Harousseau JL, Miguel JS, et al. International uniform response criteria for multiple myeloma. Leukemia. 2006;20(9): 1467-1473

20. Rajkumar SV, Harousseau JL, Durie B, et al. Consensus recommendations for the uniform reporting of clinical trials: report of the International Myeloma Workshop Consensus Panel 1. Blood. 2011;117(18): 4691-4695.

21. Xu XS, Yan X, Puchalski T, et al. Clinical implications of complex pharmacokinetics for daratumumab dose regimen in patients with relapsed/refractory multiple myeloma. Clin Pharmacol Ther. 2017:101(6):721-724.

22. Clemens PL, Yan X, Lokhorst HM, et al. Pharmacokinetics of daratumumab following intravenous infusion in relapsed or refractory multiple myeloma after prior proteasome inhibitor and immunomodulatory drug treatment. Clin Pharmacokinet. 2017;56(8):915-924.

23. Wang DD, Zhang S, Zhao H, Men AY, Parivar K. Fixed dosing versus body sizebased dosing of monoclonal antibodies in adult clinical trials. J Clin Pharmacol. 2009:49(9):1012-1024

24. Bai S, Jorga K, Xin Y, et al. A guide to rational dosing of monoclonal antibodies. Clin Pharmacokinet. 2012;51(2):119-135.

25. Mateos MV, Usmani SZ, Grosicki S, et al. Randomized, open-label, non-inferiority, phase 3 study of subcutaneous (SC) versus intravenous (IV) daratumumab (DARA) administration in patients (pts) with relapsed or refractory multiple myeloma (RRMM): body weight subgroup analysis of COLUMBA. Presented at: the 61st American Society of Hematology (ASH) Annual Meeting \& Exposition; December 7-10, 2019; Orlando, FL. Abstract 1906.

26. European Medicines Agency. MabThera: EPAR - product information; 2008.

27. European Medicines Agency. Herceptin: EPAR - product information; 2010.

28. HYOVIA [Immune Globulin Infusion 10\% (Human) With Recombinant Human Hyaluronidase] Solution for subcutaneous administration [package insert]. Westlake Village, CA: Baxalta US Inc.; 2016.

29. Palumbo A, Chanan-Khan A, Weisel K, et al. Daratumumab, bortezomib, and dexamethasone for multiple myeloma. $\mathrm{N}$ Engl J Med. 2016;375(8):754-766.

30. Dimopoulos MA, Oriol A, Nahi H, et al. Daratumumab, lenalidomide, and dexamethasone for multiple myeloma. N Engl J Med. 2016;375(14):1319-1331

31. Usmani SZ, Weiss BM, Plesner T, et al. Clinical efficacy of daratumumab monotherapy in patients with heavily pretreated relapsed or refractory multiple myeloma.
Blood. 2016;128(1):37-44.

32. Plesner T, Arkenau HT, Gimsing P, et al Phase $1 / 2$ study of daratumumab, lenalidomide, and dexamethasone for relapsed multiple myeloma. Blood. 2016;128(14):18211828.

33. Chari A, Mark TM, Krishnan A, et al. Use of montelukast to reduce infusion reactions in an early access treatment protocol of daratumumab in United States patients with relapsed or refractory multiple myeloma. Presented at: the 58th American Society of Hematology (ASH) Annual Meeting \& Exposition; December 3-6, 2016; San Diego, CA. Abstract 2142.

34. Chari A, Suvannasankha A, Fay JW, et al. Daratumumab plus pomalidomide and dexamethasone in relapsed and/or refractory multiple myeloma. Blood. 2017;130(8):974981

35. Lonial S, Weiss BM, Usmani S, et al Daratumumab monotherapy in patients with treatment-refractory multiple myeloma (SIRIUS): an open-label, randomised, phase 2 trial. Lancet. 2016:387(10027):1551-1560

36. Mateos MV, Nahi H, Legiec W, et al. Efficacy and safety of the randomized, open-label, non-inferiority, phase 3 study of subcutaneous (SC) versus intravenous (IV) daratumumab (DARA) administration in patients (pts) with relapsed or refractory multiple myeloma (RRMM): COLUMBA. Presented at: the Annual Meeting of the American Society of Clinical Oncology (ASCO); May 31-June 4, 2019; Chicago, IL. Abstract 8005.

37. Sonneveld P, Terpos E, Dimopolous M, et al. Pomalidomide and dexamethasone (pomdex) with or without daratumumab (DARA) in patients (pts) with relapsed or refractory multiple myeloma (RRMM): a multicenter randomized, phase 3 study (APOLLO). Presented at: the Annual Meeting of the American Society of Clinical Oncology (ASCO); June 1-5, 2018; Chicago, IL. Abstract TPS8059.

38. Rajkumar SV, Voorhees PM, Goldschmidt H, et al. Randomized, open-label, phase 3 study of subcutaneous daratumumab (DARA SC) versus active monitoring in patients (pts) with high-risk smoldering multiple myeloma (SMM): AQUILA. J Clin Oncol. 2018;36(15 Suppl):TPS8062.

39. Comenzo RL, Kastritis E, Mauer M, et al Subcutaneous daratumumab + cyclophosphamide, bortezomib, and dexamethasone (CyBorD) in patients with newly diagnosed amyloid light chain (AL) amyloidosis: updated safety run-in results of ANDROMEDA. Presented at: the 24th European Hematology Association (EHA) Annual Congress; June 13-16, 2019; Amsterdam, The Netherlands. Abstract S875.

40. Usmani SZ, Mateos MV, Hungria V, et al Greater treatment satisfaction in patients receiving subcutaneous versus intravenous daratumumab for relapsed or refractory multiple myeloma: COLUMBA. Presented at: the 17th International Myeloma Workshop (IMW); September 12-15, 2019; Boston MA. Abstract SP-084.

41. Chari A, San-Miguel J, McCarthy H, et al Subcutaneous (SC) daratumumab (DARA) in combination with standard multiple myeloma (MM) treatment regimens: an open-label, multicenter phase 2 study (PLEIADES) Presented at: the 17th International Myeloma Workshop (IMW); September 1215, 2019; Boston, MA. Abstract OAB-022. 